\title{
Practice Transformation Support and Patient Engagement to Improve Cardiovascular Care: From EvidenceNOW Southwest (ENSW)
}

\author{
W. Perry Dickinson, MD, Donald E. Nease, Jr., MD, Robert L. Rhyne, MD, \\ Kyle E. Knierim, MD, Douglas H. Fernald, MA, Dionisia R. de la Cerda, MPA, and \\ L. Miriam Dickinson, $\mathrm{PhD}$
}

Purpose: To improve cardiovascular care through supporting primary care practices' adoption of evidence-based guidelines.

Study Design: A cluster randomized trial compared two approaches: (1) standard practice support (practice facilitation, practice assessment with feedback, health information technology assistance, and collaborative learning sessions) and (2) standard support plus patient engagement support.

Methods: Primary outcomes were cardiovascular clinical quality measures (CQMs) collected at baseline, 9 months, and 15 months. Implementation of the first 6 "Building Blocks of High-Performing Primary Care" was assessed by practice facilitators at baseline and 3, 6, and 9 months. CQMs from practices not involved in the study served as an external comparison.

Results: A total of 211 practices completed baseline surveys. There were no differences by study arm (odds ratio [95\% confidence interval]) for aspirin use $(1.03[0.99,1.06])$, blood pressure $(0.98$ $[0.95,1.01])$, cholesterol $(0.96[0.92,1.00])$, and smoking $(1.01[0.96,1.07])$; however, there were significant improvements over time in aspirin use $(1.04[1.01,1.07])$, cholesterol $(1.05[1.03,1.08])$, and smoking $(1.03[1.01,1.06])$, but not blood pressure $(1.01[0.998,1.03])$. Improvement in enrolled practices was greater than external comparison practices across all 4 measures $($ all $P<.05)$. Implementation improved in both arms for Team-Based Care, Patient-Team Partnership, and Population Management, and improvement was greater in enhanced intervention practices (all $P<.05)$. Leadership and Data-Driven Improvement $(P<.05)$ improved significantly, with no difference by arm. A greater improvement in Building Block implementation was associated with a greater improvement in blood pressure measures $(\boldsymbol{P}<.05)$.

Conclusions: Practice transformation support can assist practices with improving quality of care. Patient engagement in practice transformation can further enhance practices' implementation of aspects of new models of care. ( $\mathrm{J}$ Am Board Fam Med 2020;33:675-686.)

Keywords: Blood Pressure, Cardiovascular Diseases, Patient Participation, Quality Improvement, Practice-Based Research, Primary Health Care, Risk Factors, Surveys and Questionnaires

\section{Introduction}

Cardiovascular disease (CVD) causes 1 in 3 deaths reported each year in the United States, with heart

This article was externally peer reviewed.

Submitted 28 October 2019; revised 6 January 2020 and 17 January 2020; accepted 23 January 2020.

From the Department of Family Medicine, University of Colorado School of Medicine, Aurora (WPD, DEN, KEK, DHF, DRdlC, LMD); Department of Family and Community Medicine, University of New Mexico School of Medicine, Albuquerque (RLR).

Funding: This project was supported by the Agency for Healthcare Research and Quality grant \#1R18HS023904. disease the first and stroke the fifth leading cause of death. ${ }^{1-3}$ Addressing CVD risk factors can greatly reduce the burden of CVD. Primary care practices must transform to deliver a higher level of evidence-based prevention to decrease cardiovascular risk. Interventions that emphasize patient-centered

Conflicting and competing interests: All authors state that they have no competing interests.

Corresponding author: W. Perry Dickinson, MD, University of Colorado School of Medicine, Department of Family Medicine, 12631 E. 17th Ave., Mail Stop F496, Aurora, CO 80045-0508 (E-mail: perry.dickinson@cuanschutz.edu). 
care have been shown to be effective. ${ }^{4-11}$ Practices often require assistance integrating new practice approaches into clinical operations. Practice facilitation can enhance implementation of new programs for patients with chronic disease. ${ }^{12-16}$ Other methods of practice support, including academic detailing, collaborative learning sessions, and health information technology (HIT) assistance, have also been shown to be effective. ${ }^{12,17-28}$

Practices are increasingly including patients as part of practice transformation through the creation of patient and family advisory councils and participation in quality improvement teams. ${ }^{29-33}$ The boot camp translation method can inform implementation of evidence-based care through engaging patients, clinicians, and staff members to translate best practices into culturally and community-relevant messages and materials for patient engagement. ${ }^{26,34-38}$ Although patient engagement through patient and family advisory councils has been encouraged as part of practice transformation, no study to date has compared the impact of adding patient engagement methods to more standard practice transformation methods.

EvidenceNOW Southwest (ENSW), a collaborative effort between Colorado and New Mexico, is 1 of 7 regional cooperatives funded by the Agency for Health care Research and Quality to help small-and medium-sized primary care practices improve cardiovascular care while also improving practice capacity for quality improvement. The conceptual model for the practice transformation support design, adapted and modified from previous models, ${ }^{39,40}$ can be found in Figure 1. Based on this model, we developed 2 practice transformation support interventions: (1) "standard" practice transformation support, including practice facilitation, HIT assistance, and collaborative learning sessions; and (2) an "enhanced" approach that added support for patient engagement (including patient and family advisory councils and patient engagement materials tailored for specific populations through boot camp translation) to the standard intervention. We hypothesized that both intervention arms would demonstrate improvement in cardiovascular risk outcomes, that the addition of patient engagement activities would result in a greater improvement for the enhanced intervention, and that both approaches would result in greater improvements compared with an external comparison group receiving no support. The "Bodenheimer Building Blocks for HighPerforming Primary Care" ${ }^{\text {} 41}$ were used as a framework for guiding practice improvement and were hypothesized as key intermediate outcomes and mediators of the practice transformation interventions.

\section{Methods \\ Design}

The project was approved by the Colorado Multiple Institutional Review Board and the University of New Mexico Human Research Protections Office and is registered on ClinicalTrials.gov (NCT02515578, protocol identifier 15-0403). ENSW is a cluster randomized trial with 2 active interventions and an external comparison group that received no intervention. As we compared 2 active intervention arms and there were ongoing national initiatives focused on cardiovascular care (eg, Million Hearts), the external comparison group was included to control for temporal trend. Because of the intent to implement community engagement interventions at the community and regional level and the timeline of the study, which required knowing which regions were in which study arm before completing practice recruitment, we used geographic-based covariate constrained randomization to allocate geographicbased regions (26 in Colorado, 16 in New Mexico) to

\section{Figure 1. Conceptual model for EvidenceNOW Southwest intervention.}

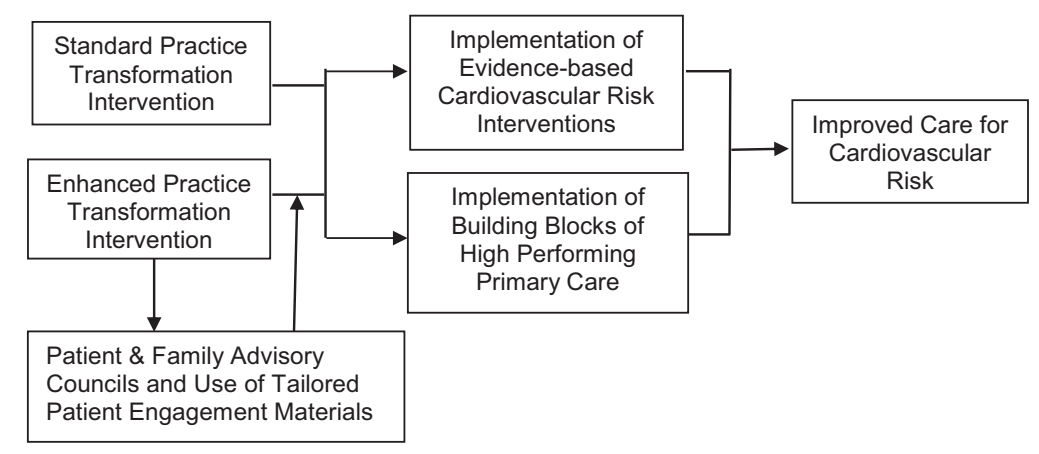


intervention arms to achieve balanced study arms with respect to underlying populations and resources. This approach is described in detail elsewhere. ${ }^{38}$ This study was powered to detect a 0.47 effect size difference between study arms on practice-level outcomes with no clustering (ie, single measure per practice) or a medium linear trend effect (differences increase from 0 to $.5 \mathrm{SD}$ over time). ${ }^{42}$ An external cohort of similar practices was obtained from the DARTNet Institute, a nonprofit institute that coordinates and supports research, quality improvement, and safety activities across multiple research networks through the collection of electronic health data. ${ }^{38,43}$

\section{Interventions}

As detailed below and summarized in Table 1, we compared the effectiveness of a standard practice transformation support intervention to practice transformation support enhanced by patient and community engagement. Twenty "practice transformation organizations" (PTOs) across Colorado and New Mexico provided the practice facilitator and clinical HIT advisor (CHITA) services for the practices. Standardized training was provided by the research team to experienced practice facilitators and clinical HIT advisors from the participating PTOs in a group setting. In some cases, the same individual served in both practice facilitator and CHITA roles, depending on individual skills and PTO resources. Required field notes tracked contacts and content of all facilitator and CHITA activities and were reviewed regularly by the research team to ensure fidelity to the intervention components.

\section{All practices, standard and enhanced, received the following}

- Practice assessment with feedback to practice. A baseline practice survey assessed practice culture and change capacity, recent or ongoing practice transformation efforts, and current level of implementation of Patient-Centered Medical Home (PCMH) components and cardiovascular care. Assessments were aligned across EvidenceNOW cooperatives. Results of assessments were summarized in feedback reports to practices to help initiate the quality improvement process through a reflective discussion of practices' needs and priorities.

- Practice facilitation with quality improvement team meetings. Active practice facilitation (and the intervention baseline) began with the feedback report to the practice. The active facilitation phase focused on monthly meetings of a practice improvement team, consisting of diverse representatives of the practice (eg, front office, medical assistants, and clinicians). The facilitator helped the team select quality improvement activities to improve care for patients with cardiovascular risk and then assisted the team in using data and quality improvement methods for rapid cycle change. The goals were to implement practice transformation activities based on the first six Building Blocks and evidence-based cardiovascular risk interventions. Practice facilitation lasted for 9 months.

- Collaborative learning sessions. Representatives from each practice participated in 2 collaborative learning sessions during the course of their intervention, providing an opportunity to share lessons learned with other practices working on the same interventions.

- HIT support. On-the-ground HIT assistance by CHITAs aimed at developing data capacity for quality measures and population management and linkage to our quality measure data aggregation system. CHITAs met with practices approximately monthly during the 9-month intervention. CHITAs supported quarterly clinical quality measure (CQM) reporting and provided measure feedback reports to the practices.

- Cardiovascular toolkit. An online hub of support materials and e-learning modules aligning with

Table 1. EvidenceNow Southwest Transformation Support Interventions for Standard and Enhanced Arm Practices

\begin{tabular}{lcc}
\hline Program Element & Standard Practice Support & Enhanced with Patient Engagement \\
\hline Practice assessment with feedback to practice & Yes & Yes \\
Practice facilitation with monthly improvement team meetings & Yes & Yes \\
Collaborative learning sessions & Yes & Yes \\
Health Information Technology support & Yes & Yes \\
Cardiovascular toolkit, including e-learning modules & Yes & Yes \\
Patient engagement materials from boot camp translation & No & Yes \\
Support for Patient and Family Advisory Councils & No & Yes \\
\hline
\end{tabular}


the Million Hearts Campaign ${ }^{44}$ and the first six Building Blocks were offered to practices, focusing on the following areas: implementing evidence-based guidelines for aspirin therapy and treatment of hypertension (HTN) and cholesterol; promoting systems to support self-management, such as telephone follow-up and home blood pressure monitoring; linking primary care practices and community and public health resources; implementing multidisciplinary teambased care; and implementing a comprehensive approach to smoking cessation, including referrals to community resources.

\section{Enhanced intervention practices received 2 additional elements}

- Regionally-tailored patient engagement materials. Boot camp translation was used to produce patient engagement materials based on practice and patient input regarding the local context. Within regions randomized to the patient engagement arm, we convened 4 regional groups of 16 or fewer individuals representing patients, clinicians, staff members, and local public health. This process and examples of the locally developed materials are described elsewhere. ${ }^{38}$ The patient engagement materials developed through this process were offered only to the enhanced arm practices.

- Patient and family advisory councils. ENSW provided centralized resources and trained the practice facilitators to support the formation and active use of patient and family advisory councils. Notably, e-learning modules were developed to assist practices in engaging patients in the practice transformation process.

\section{Measures}

\section{Implementation}

The conceptual and operational framework for the practice facilitation intervention was based on the first 6 Building Blocks for High-Performing Primary Care. ${ }^{41}$ This was assessed using an Implementation Tracker in which practice facilitators rated practices' progress on the 6 building blocks (no activity, in progress, or complete) at baseline and 3, 6, and 9 months (active intervention period). Building Blocks were scored as sum of items (reversed, if necessary), scaled from 0 to 100 . Cronbach's $\alpha$ for items within each subscale ranged from 0.79 to 0.92 .

Primary effectiveness outcomes (ABCS measures) were obtained from practice-level CQMs on cardiovascular risk management (aspirin use, blood pressure, cholesterol, and smoking) collected quarterly. Each measure was based on numerators and denominators from the previous 12 months. Quality assurance strategies were imposed for all measures, and any data points that deviated from expected values were verified and corrected, if necessary, by the data manager and the CHITA. Measures from the DARTNet external comparison practices were used as reported to the research team after applying the quality restrictions used for ENSW practices. The ABCS measures are described below, as follows:

- Aspirin use. Percent of patients 18 years and older with ischemic vascular disease (IVD) with documented use of aspirin or other antithrombotic. ${ }^{45}$

- Blood pressure management. Percent of patients aged 18-85 who had a diagnosis of HTN and whose blood pressure was adequately controlled $(<140 / 90)$ during the measurement year. ${ }^{46}$

- Cholesterol management. Percent of patients from three categories considered at high risk for CVD who were prescribed or were on statin therapy. ${ }^{47}$

- Smoking cessation support. Percent of patients aged 18 years or older screened for tobacco use one or more times within 24 months and who received cessation counseling intervention if identified as a tobacco user. $^{48}$

A team or an individual designated by the practice completed a practice survey at baseline that included key practice demographic information.

\section{Data Analysis}

Descriptive statistics were computed for baseline practice characteristics, initially testing for differences between: (1) different intervention arms and (2) practice dropouts versus nondropouts. For analyses of differences between study arms, practice-level covariates were screened in bivariate analyses and initially included in multivariate analysis if they were related to the outcome at $P<.1$, differed between treatment arms, or were associated with dropout. When there was high collinearity among covariates, a single covariate was chosen to represent that domain. In general, we used intent-to-treat analyses that used all available data, assuming ignorable missingness. ${ }^{49,50}$ Because normality assumptions were not met for the CQMs, we used generalized linear mixed models (mixed effects beta regression) with random intercepts for practice to analyze the data. This approach has been shown to be more suitable than linear mixed models for continuous data that is 
bounded $(0,1)$ and often skewed, such as proportions. ${ }^{51-53}$ To include all valid observations, a small constant (0.001) was added or subtracted from observations that had a value of 0 or 1 . Building Block outcomes were analyzed using general linear mixed models with a random effect for practice. We assessed overall improvement over time (main effect for time) as well as differential improvement by study arm or ENSW vs external comparison group (time by group interaction term) for all analyses. In addition, we examined improvement in Building Blocks of High-Performing Primary Care (secondary/intermediate outcomes) as potential mediators of improvement in ABCS quality measures (see conceptual model) using general linear mixed models. ${ }^{54,55}$ Hypothesis tests were 2-sided with $\alpha=0.05$ or $\mathrm{p}$ values reported. All statistical analyses were performed using SAS version 9.4 (SAS Institute Inc., Cary, NC).

\section{External comparison group}

A total of 522 practices in the database provided by DARTNet Institute had complete data on practice ownership (FQHC, private, and hospital) and location (rural/urban), along with county-level census data on population demographics (\% over age 65 , $\%$ Hispanic, \% black, \% female, and median household income). From the subset of 457 practices that had nonzero proportions on at least 3 of the 4 CQMs during the baseline 12-month performance period ending December 31, 2016, we selected a random sample of 200 practices that was comparable to ENSW practices. Adapting our covariate constrained randomization procedure, ${ }^{56}$ we (1) generated 1,000 random samples of 200 practices from the 457 eligible practices in the DARTNet database, (2) calculated a balance criterion for external comparison group versus ENSW (sum of the absolute values of the standardized distances between means of each random sample and the overall mean from ENSW practices on the above variables), (3) identified a candidate set of random samples as the top $30 \%$ overall that had at least $14.5 \%$ rural (a total of 50 random samples of 200 practices), and 4) randomly selected a single random sample of 200 practices from the candidate set as the external comparison group. Data for the comparison group were extracted and processed in mid-2017. Analyses comparing active intervention practices (all ENSW practices) to the external comparison group used similar approaches to those described above.

\section{Results}

\section{Practice Engagement}

As described previously, ${ }^{38} 26$ regions in Colorado and 16 regions in New Mexico were randomized to standard versus intervention study arms. A total of 312 practices were initially given information about the study and invited to submit a practice enrollment application (Figure 2). A total of 239 practices completed initial enrollment for the project; 211 actually began the intervention and completed baseline surveys. Twelve practices dropped during the intervention; 199 practices completed the 9-month interventions. Characteristics of practices that dropped out were similar to those that remained (ie, not significantly different, all $P>.15$ ). Despite using methods to assure balance on population characteristics, ${ }^{38}$ study arms differed somewhat (Table 2), largely reflecting the higher percentage of FQHC and rural practices in the standard intervention arm. The external comparison group also differed somewhat from ENSW practices. Among external comparison practices, $68.5 \%$ were clinician owned, 25.5\% hospital owned, and 6\% FQHCs; $15.5 \%$ were in rural areas. On average, DARTNet practices had 2.6 full-time equivalent clinicians and were located in areas with 7.6\% Hispanic and 5.5\% black populations.

\section{CQMs}

Table 3 shows results of the adjusted analyses of the ABCS measures over time for standard versus enhanced intervention arms, including odds ratios with $95 \%$ confidence intervals from the beta regression models. Change over time did not differ by study arm for any CQM (Table 2) (aspirin use, $P=.114$; blood pressure, $P=.178$; cholesterol, $P=.078$; smoking cessation, $P=.596$ ). In the absence of significant differential change, study arms were combined to assess overall improvement, which was significant for aspirin use (odds ratio [95\% confidence interval]) $(1.04[1.01,1.07], P<.001)$, cholesterol composite $(1.05[1.03,1.08], P<.001)$, and smoking $(1.03[1.01,1.06], P=.014)$, but not blood pressure control $(1.01[0.998,1.03], P=.084)$.

Because practices were allowed to report cholesterol measures for subpopulations of patients instead of the composite measure, additional analyses were performed for these measures. Subpopulations included atherosclerotic CVD (ASCVD) $(\mathrm{n}=109)$, LDL $>190(n=36)$, and diabetes $(n=93)$. Analyses 
Figure 2. Consolidated Standards of Reporting Trials (CONSORT) practice engagement diagram.

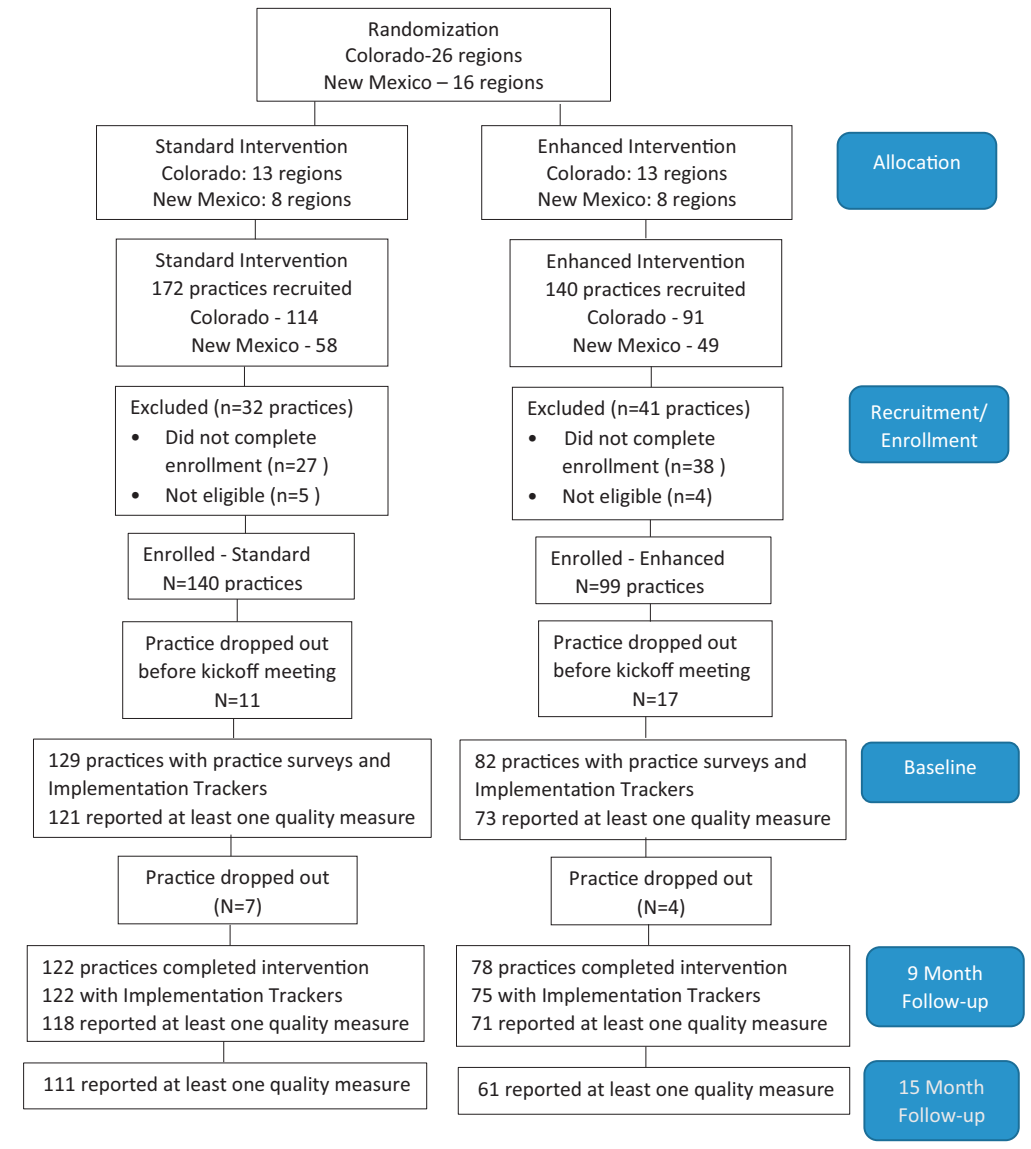

of subpopulation measures showed overall improvement for ASCVD $(P=.009)$ and LDL subpopulations $(P=.004)$ and marginal improvement for diabetes $(P=.077)$.

\section{Comparison to External Comparison Group}

To better understand whether improvement in ENSW practices might be due to temporal trends in cardiovascular care, ENSW practices were compared with the external comparison group on ABCS measures over time. Even after using procedures to maximize comparability between comparison practices and ENSW practices, there were differences in baseline levels of the ABCS measures, with ENSW practices having higher (much higher for aspirin use) values on all ABCS measures than the external comparison group. Baseline and 12month follow-up estimates for ENSW and external comparison practices, back-transformed to represent actual proportions and adjusted for rural location and practice type, are shown in Table 4. Compared with external comparison practices,
ENSW practices demonstrated greater improvement on all 4 ABCS measures, including blood pressure (all $P<.05)$.

\section{Implementation of the Building Blocks for High- Performing Primary Care}

The Building Blocks constituted an important intermediate outcome for both practice transformation interventions; we also hypothesized that improvement in certain key Building Blocks (especially Patient-Team Partnership) would be greater in the enhanced intervention arm. Table 5 shows results of the unadjusted and adjusted analyses of Implementation Tracker scores over time. When they were adjusted for practice type and rural location, there was a significant overall improvement in Team-Based Care, Patient Team Partnership, and Population Management (all $P<.001$, as indicated by improvement in the standard group in Table 5), and improvement was greater in enhanced intervention practices (all $P<.05$, indicated by differential improvement in the enhanced group in Table 
Table 2. EvidenceNow Southwest Baseline Practice Characteristics

\begin{tabular}{|c|c|c|c|c|}
\hline \multirow[b]{2}{*}{ Characteristic } & \multicolumn{3}{|c|}{ Mean (SD) or \% of: } & \multirow{2}{*}{$\begin{array}{c}\text { Standard vs } \\
\text { Enhanced } P \text { Value }\end{array}$} \\
\hline & Total $(\mathrm{N}=211)$ & Standard $(\mathrm{N}=129)$ & Enhanced $(\mathrm{N}=82)$ & \\
\hline \multicolumn{5}{|l|}{ State } \\
\hline Colorado & $74.9 \%$ & $69.8 \%$ & $82.9 \%$ & .032 \\
\hline New Mexico & $25.1 \%$ & $30.2 \%$ & $17.1 \%$ & \\
\hline \multicolumn{5}{|l|}{ Ownership } \\
\hline Clinician & $47.9 \%$ & $32.6 \%$ & $72.0 \%$ & \\
\hline Hospital/academic center & $15.6 \%$ & $14.7 \%$ & $17.1 \%$ & $<.001$ \\
\hline Federally Qualified Health Centers & $36.5 \%$ & $52.7 \%$ & $11.0 \%$ & \\
\hline Rural & $28.9 \%$ & $37.2 \%$ & $15.9 \%$ & .001 \\
\hline Patient-Centered Medical Home & $44.6 \%$ & $55.8 \%$ & $26.8 \%$ & $<.001$ \\
\hline \multicolumn{5}{|l|}{ Practice size, number of providers } \\
\hline Solo & $21.0 \%$ & $24.2 \%$ & $16.1 \%$ & \\
\hline $2-5$ & $60.5 \%$ & $60.5 \%$ & $60.5 \%$ & .348 \\
\hline $6-10$ & $16.6 \%$ & $13.7 \%$ & $21.0 \%$ & \\
\hline$>10$ & $2.0 \%$ & $1.6 \%$ & $2.5 \%$ & \\
\hline \multicolumn{5}{|l|}{ Payer mix } \\
\hline \% Medicaid & $27.4(22.1)$ & $31.8(20.5)$ & $19.9(22.9)$ & $<.001$ \\
\hline$\%$ Medicare & $18.7(14.0)$ & $18.7(13.2)$ & $18.8(15.4)$ & .988 \\
\hline$\%$ No insurance & $11.2(13.9)$ & $12.3(13.1)$ & $9.2(15.0)$ & .142 \\
\hline$\%$ Commercial insurance & $37.6(25.2)$ & $32.5(22.3)$ & $46.0(27.7)$ & $<.001$ \\
\hline \multicolumn{5}{|l|}{ Demographics } \\
\hline \multicolumn{5}{|l|}{ Race } \\
\hline \% Black & $3.5(5.6)$ & $2.9(5.7)$ & $4.5(5.5)$ & .054 \\
\hline$\%$ Asian & $2.6(6.3)$ & $2.2(5.2)$ & $3.2(7.7)$ & .322 \\
\hline $\begin{array}{l}\text { \% Alaskan Native/American } \\
\text { Indian }\end{array}$ & $3.6(13.6)$ & $5.0(17.1)$ & $1.2(2.7)$ & .021 \\
\hline \multicolumn{5}{|l|}{ Ethnicity } \\
\hline \% Hispanic & $29.4(25.0)$ & $34.7(26.1)$ & $20.6(20.5)$ & $<.001$ \\
\hline \multicolumn{5}{|l|}{ Gender } \\
\hline \% Female & $54.2(9.3)$ & $53.6(10.0)$ & $55.1(8.0)$ & .277 \\
\hline \multicolumn{5}{|l|}{ Patient registries } \\
\hline Any registry & $67.8 \%$ & $70.5 \%$ & $63.4 \%$ & .280 \\
\hline Ischemic vascular disease & $37.9 \%$ & $42.6 \%$ & $30.5 \%$ & .076 \\
\hline Hypertension & $54.0 \%$ & $59.7 \%$ & $45.1 \%$ & .039 \\
\hline High cholesterol & $44.6 \%$ & $52.7 \%$ & $31.7 \%$ & .003 \\
\hline Diabetes & $64.0 \%$ & $65.9 \%$ & $61.0 \%$ & .468 \\
\hline Prevention services & $53.6 \%$ & $62.8 \%$ & $39.0 \%$ & .001 \\
\hline High risk or utilization patients & $35.1 \%$ & $38.0 \%$ & $30.5 \%$ & .266 \\
\hline \multicolumn{5}{|l|}{ Use of clinical guidelines } \\
\hline \multicolumn{5}{|l|}{ Prevention } \\
\hline Number of guidelines & $1.8(1.4)$ & $1.9(1.4)$ & $1.7(1.3)$ & .276 \\
\hline No guidelines used & $16.6 \%$ & $16.3 \%$ & $17.1 \%$ & .880 \\
\hline Informal (agreed or posted) & $62.1 \%$ & $58.1 \%$ & $68.3 \%$ & .138 \\
\hline Standing orders & $33.7 \%$ & $38.8 \%$ & $25.6 \%$ & .049 \\
\hline Electronic health record prompts & $59.7 \%$ & $62.0 \%$ & $56.1 \%$ & .393 \\
\hline \multicolumn{5}{|l|}{ Management } \\
\hline Number of guidelines & $1.7(1.4)$ & $1.8(1.4)$ & $1.6(1.3)$ & .354 \\
\hline No guidelines used & $18.5 \%$ & $18.6 \%$ & $18.3 \%$ & .955 \\
\hline Informal (agreed or posted) & $59.7 \%$ & $56.6 \%$ & $64.6 \%$ & .246 \\
\hline Standing orders & $29.9 \%$ & $34.1 \%$ & $23.2 \%$ & .091 \\
\hline Electronic health record prompts & $56.4 \%$ & $58.1 \%$ & $53.7 \%$ & .522 \\
\hline
\end{tabular}

$\mathrm{SD}$, standard deviation. 
Table 3. ABCS Clinical Quality Measures: Practice Estimate Comparisons between Study Arms

\begin{tabular}{lcccc}
\hline & \multicolumn{4}{c}{ Adjusted OR (95\% CI) for: } \\
\cline { 2 - 5 } Variable & $\begin{array}{c}\text { Aspirin Use } \\
(\mathrm{N}=179)\end{array}$ & $\begin{array}{c}\text { Blood Pressure } \\
(\mathrm{N}=189)\end{array}$ & $\begin{array}{c}\text { Cholesterol } \\
(\mathrm{N}=90)\end{array}$ & $\begin{array}{c}\text { Smoking } \\
(\mathrm{N}=183)\end{array}$ \\
\hline Time (change per quarter in standard arm) & $1.03(1.01,1.05)$ & $1.02(1.00,1.04)$ & $1.07(1.04,1.09)$ & $1.03(0.99,1.06)$ \\
Baseline differences (enhanced vs standard) & $0.85(0.59,1.22)$ & $1.03(0.78,1.36)$ & $1.07(0.80,1.43)$ & $0.79(0.44,1.44)$ \\
Clinician-owned (ref) & & & & \\
Hospital Owned & $2.85(1.83,4.44)$ & $0.62(0.44,0.88)$ & $1.18(0.88,1.57)$ & $0.50(0.24,1.02)$ \\
Federally Qualified Health Center & $1.62(1.12,2.34)$ & $0.94(0.71,1.26)$ & $0.84(0.63,1.15)$ & $2.37(1.28,4.41)$ \\
Time by intervention & $1.03(0.99,1.06)$ & $0.98(0.95,1.01)$ & $0.96(0.92,1.00)$ & $1.01(0.96,1.07)$ \\
\hline
\end{tabular}

CI, confidence interval; OR, odds ratio.

5). There was also significant overall improvement in Leadership and Data-Driven Improvement (both $P<.001)$, but improvement did not differ between standard and enhanced practices (both $P>.3$ ).

\section{Mediation}

Based on the conceptual model and the framework for the Practice Transformation interventions, we hypothesized that more successful implementation of the Building Blocks would, in turn, result in improved delivery of cardiovascular care. To explore these relationships, we examined the association between change from baseline in the Building Blocks during the active 9-month intervention period and change from baseline in the ABCS CQMs at 9 to 15 months in a mediational analysis. Improvements in the overall Building Blocks (overall mean score), Data-Driven Improvement, Team-Based Care, and Population Management were significantly associated with improvement in blood pressure CQMs. Coefficients represent additional improvement in CQMs per 10point increase in Building Block scores (coeff [SE]) (overall Building Blocks, 0.0128 [0.0054], $P=.019$; Data-Driven Improvement, 0.0010 [0.0004], $P=.023$;
Team-Based Care; 0.0102 [0.0041], $P=.013$; Population Management, 0.0109 [0.0037], $P=.003)$. Improvement in Population Management was also significantly associated with improvement in aspirin use CQMs (0.0074 [0.0036], $P=.0415)$. Improvement in Building Blocks was not significantly associated with cholesterol or smoking CQMs (all $P>.05$ ).

\section{Discussion}

Practice transformation support using practice facilitation, clinical HIT support, and collaborative learning sessions resulted in primary care practices improving quality of care by implementing elements of the Building Blocks of High-Performing Primary Care and evidence-based interventions to reduce cardiovascular risk. Adding support for implementing patient and family advisory councils and other forms of patient engagement in quality improvement efforts increased practice implementation of various Building Blocks but was not associated with differential improvement on the ABCS CQMs. The overall intervention yielded improvements in important CVD risk reduction metrics, especially when compared with the external comparison group.

Table 4. ABCS Clinical Quality Measures: Practice Estimate Comparisons between Combined Study Arms and External Controls

\begin{tabular}{|c|c|c|c|c|c|}
\hline \multirow[b]{2}{*}{ Measure } & \multicolumn{2}{|c|}{ ENSW Practices (Est [95\% CI]) } & \multicolumn{2}{|c|}{ External Controls (Est [95\% CI]) } & \multirow{2}{*}{$\begin{array}{c}P \text { Value for } \\
\text { Difference } \\
\text { in Slopes }\end{array}$} \\
\hline & Baseline & 12 Months & Baseline & 12 Months & \\
\hline Aspirin & $0.668(0.628,0.705)$ & $0.763(0.722,0.799)$ & $0.049(0.039,0.061)$ & $0.049(0.036,0.068)$ & .012 \\
\hline Blood Pressure & $0.606(0.572,0.639)$ & $0.618(0.585,0.650)$ & $0.441(0.402,0.481)$ & $0.429(0.392,0.467)$ & .040 \\
\hline Smoking & $0.826(0.794,0.854)$ & $0.843(0.814,0.868)$ & $0.167(0.138,0.201)$ & $0.144(0.120,0.177)$ & $<.001$ \\
\hline Cholesterol & $0.659(0.628,0.689)$ & $0.704(0.675,0.730)$ & $0.511(0.481,0.540)$ & $0.494(0.465,0.423)$ & $<.001$ \\
\hline
\end{tabular}

Est, estimate; ENSW, EvidenceNOW Southwest; CI, confidence interval. 
Table 5. Implementation of First Six Building Blocks of High-Performing Primary Care

\begin{tabular}{llccc}
\hline Building Block & Term & Standard Estimate $^{*}(\mathrm{SE})$ & Enhanced Estimate $^{*}(\mathrm{SE})$ & Comparison and $P$ Value \\
\hline \multirow{2}{*}{ Leadership } & Baseline & $51.9(2.1)$ & $56.5(2.7)$ & Overall improvement, $<.001$ \\
& Follow-up & $82.2(2.1)$ & $87.8(2.8)$ & Time $\times$ arm, .783 \\
Data-Driven Improvement & Baseline & $38.2(2.2)$ & $38.5(2.8)$ & Overall improvement, $<.001$ \\
& Follow-up & $76.2(2.2)$ & $80.4(2.9)$ & Time $\times$ arm, .327 \\
Empanelment & Baseline & $60.0(3.5)$ & $49.5(4.6)$ & Overall improvement, .202 \\
& Follow-up & $55.9(3.6)$ & $55.6(4.7)$ & Time $\times$ arm, .052 \\
Team-Based Care & Baseline & $49.4(2.6)$ & $48.8(3.3)$ & Overall improvement, $<.001$ \\
& Follow-up & $65.3(2.6)$ & $72.2(3.4)$ & Time $\times$ arm, .043 \\
Patient-Team Partnership & Baseline & $30.81(1.8)$ & $27.7(2.3)$ & Overall improvement, $<.001$ \\
& Follow-up & $44.4(1.8)$ & $46.3(2.3)$ & Time $\times$ arm, .011 \\
Population Management & Baseline & $40.3(2.4)$ & $40.3(3.2)$ & Overall improvement, $<.001$ \\
& Follow-up & $60.5(2.5)$ & $63.6(3.3)$ & Time $\times$ arm, .033 \\
\hline
\end{tabular}

*Adjusted for practice ownership and rural location.

Patient engagement in practice improvement and redesign projects has been encouraged as a method for making care more patient centered. This project provides evidence that practices respond to support for patient engagement activities and that the resulting patient involvement can improve practice implementation of elements of high-performing primary care. Although there were not significant differences between the study groups in CQM improvements, the 9-month intervention provided a limited time for practices to implement a fully functional patient and family advisory council and then to see the impact of the council on outcomes. This is particularly true for CQMs, such as blood pressure control, that generally have a lag between implementation of changes in care and improvements in the measures, and this could be partially responsible for the lack of significant differences between the 2 groups on ABCS measure improvement. It also is possible that the patient and family advisory councils focused practice attention on improvements not directly tied to the ABCS CQMs. It should be noted that the study design did not exclude practices that had pre-existing patient and family advisory councils from the standard intervention group, and this may have attenuated differences in outcomes between the 2 groups.

Mediator analyses showed that increased implementation of the first 6 Building Blocks of HighPerforming Primary Care served as mediators for improvements in blood pressure control and, to a lesser extent, aspirin use CQMs. This is particularly interesting in that the blood pressure control measure is the only 1 of the ABCS measures directly tied to clinical outcomes, with the other 3 measure tied to process of care. Process of care measures are often easier to change than clinical outcomes, in part because (1) improvements in data entry and capture can result in major improvements and (2) changes in process are more immediately reflected in the measure. This can been seen in our results, in which blood pressure did not improve as impressively as the process of care measures. However, for a clinical outcome such as blood pressure control that is difficult to change, the implementation of the Building Blocks might be particularly important in producing improvements, whereas the Building Blocks may be less important to produce changes in process of care measures. This will be interesting to explore in future projects.

This study adds to the growing evidence for the effectiveness of practice facilitation as a method for improving implementation of evidence-based interventions. ENSW results are consistent with other research demonstrating that practice facilitation can assist practices in improvements in cardiovascular care. ${ }^{16}$ In addition, this study shows that practice facilitation can induce practices to take up important patient engagement activities. The intervention period for this project was relatively short for the level of practice transformation work being targeted. The study was further complicated by major changes in the cholesterol treatment guidelines just as the project was starting. Difficulties and delays with obtaining clean CQM data as reported elsewhere ${ }^{57,58}$ made 
quality improvement efforts focused on specific measures problematic as well. Despite those barriers and limitations, practices improved all 4 of the ABCS measures significantly when compared with an external comparison group. Furthermore, they showed significant improvement in their implementation of many advanced primary care activities.

There are other potential limitations beyond the limited intervention and follow-up periods. Although a broad range of practice types across Colorado and New Mexico were engaged, they may not be representative of all primary care practices. Similarly, the external comparison group, drawn from a pool of practices involved in activities with research, safety, and quality activities, differed somewhat from ENSW practices and may not provide a representative comparison population. CQMs from comparison practices, which were obtained via data extraction from electronic health records, were substantially lower than ENSW practices for all 4 CQMs. The lower rates for external comparison practices may be due to lower ascertainment, lack of onsite HIT support for practices, and/or absence of focus specifically on ABCS measures. However, although some measures are quite low in comparison practices, the trajectories should be a reasonable reflection of change over time because all patient-level electronic health record data from the entire time frame (from which these measures were generated) were obtained and processed from a single data extraction in each comparison practice and processed using the same methods. Randomization of geographic regions (rather than practices) was necessary before recruiting practices to conduct regional boot camp translation activities, and although this was not ideal, it was necessary to conduct the study, as discussed elsewhere. ${ }^{38}$ Because multiple PTOs provided the practice facilitation support, there could be underlying relationships between practice demographic characteristics and individual PTOs, so that differences seen for practice types could be due to PTO performance rather than the practice characteristics. However, results of sensitivity analyses adjusted for PTO were very similar.

With multiple ongoing and upcoming efforts underway to expand primary care capacity, investment in supporting practices through practice facilitation, clinical HIT support, and other forms of practice transformation support should be considered. Patient engagement in practice transformation efforts seems effective for improving key process of care outcomes, although further studies are needed.
To see this article online, please go to: http://jabfm.org/content/ 33/5/675.full.

\section{References}

1. Go AS, Mozaffarian D, Roger VL, et al. Heart disease and stroke statistics-2014 update: a report from the American Heart Association. Circulation 2014;129:e28-e292.

2. Murphy SL, Kochanek KD, Xu JQ, Arias E. Mortality in the United States, 2014. NCHS data brief, no 229; https://www.cdc.gov/nchs/data/databriefs/db229.pdf. Published 2015. Accessed September 10, 2019.

3. Mozaffarian D, Benjamin EJ, Go AS, et al. Heart disease and stroke statistics-2016 update: a report from the American Heart Association. Circulation 2016;133:e38-360.

4. Wagner EH, Austin BT, Von Korff M. Organizing care for patients with chronic illness. Milbank Q 1996;74:511-544.

5. Fjeldsoe B, Neuhaus M, Winkler E, Eakin E. Systematic review of maintenance of behavior change following physical activity and dietary interventions. Health Psychol 2011;30:99-109.

6. Greaves CJ, Sheppard KE, Abraham C, et al. Systematic review of reviews of intervention components associated with increased effectiveness in dietary and physical activity interventions. BMC Public Health. 2011;11:119.

7. Huff LS, Zittleman L, DeAlleaume L, et al. What keeps patients from adhering to a home blood pressure program? J Am Board Fam Med 2011;24:370-9.

8. Uhlig K, Patel K, Ip S, Kitsios GD, Balk EM. Selfmeasured blood pressure monitoring in the management of hypertension: a systematic review and meta-analysis. Ann Intern Med 2013;159:185-94.

9. Schroeder K, Fahey T, Ebrahim S. Interventions for improving adherence to treatment in patients with high blood pressure in ambulatory settings. Cochrane Database Syst Rev 2004;CD004804.

10. Viera AJ, Jamieson B, Dealleaume L. Clinical inquiries. How effective are hypertension self-care interventions? J Fam Pract 2007;56:229-31.

11. Proia KK, Thota AB, Njie GJ, et al. Team-based care and improved blood pressure control. Am J Prev Med 2015;49:772-83.

12. Dickinson WP, Dickinson LM, Nutting PA, et al. Practice facilitation to improve diabetes care in primary care: a report from the EPIC randomized clinical trial. Ann Fam Med 2014;12:8-16.

13. Parchman ML, Noel PH, Culler SD, et al. A randomized trial of practice facilitation to improve the delivery of chronic illness care in primary care: initial and sustained effects. Implement Sci 2013;8:93.

14. Nagykaldi Z, Mold JW, Aspy CB. Practice facilitators: a review of the literature. Fam Med 2005;37:581-8.

15. Baskerville NB, Liddy C, Hogg W. Systematic review and meta-analysis of practice facilitation 
within primary care settings. Ann Fam Med 2012;10:63-74.

16. Wang A, Pollack T, Kadziel LA, et al. Impact of practice facilitation in primary care on chronic disease care processes and outcomes: a systematic review. J Gen Intern Med 2018;33:1968-77.

17. Wagner EH, Glasgow RE, Davis C, et al. Quality improvement in chronic illness care: a collaborative approach. Jt Comm J Qual Improv 2001;27:63-80.

18. Stange KC, Goodwin MA, Zyzanski SJ, Dietrich AJ. Sustainability of a practice-individualized preventive service delivery intervention. Am J Prev Med 2003;25:296-300.

19. Hogg W, Baskerville N, Nykiforuk C, Mallen D. Improved preventive care in family practices with outreach facilitation: understanding success and failure. J Health Serv Res Policy 2002;7:195-201.

20. Ruhe MC, Weyer SM, Zronek S, Wilkinson A, Wilkinson PS, Stange KC. Facilitating practice change: Lessons from the STEP-UP clinical trial. Prev Med 2005;40:729-34.

21. Morrow RW, Gooding AD, Clark C. Improving physicians' preventive health care behavior through peer review and financial incentives. Arch Fam Med 1995;4:165-9.

22. Grumbach $\mathrm{K}$, Bainbridge $\mathrm{E}$, Bodenheimer $\mathrm{T}$. Facilitating improvement in primary care: the promise of practice coaching. Publication 1605. Commonwealth Fund, New York, NY; 2012.

23. Pearson ML, Wu S, Schaefer J, et al. Assessing the implementation of the chronic care model in quality improvement collaboratives. Health Serv Res 2005;40:978-96.

24. Mittman BS. Creating the evidence base for quality improvement collaboratives. Ann Intern Med 2004;140:897-901.

25. Ovretveit J, Bate P, Cleary P, et al. Quality collaboratives: lessons from research. Qual Saf Health Care 2002;11:345-51.

26. Bender BG, Dickinson P, Rankin A, Wamboldt FS, Zittleman L, Westfall JM. The Colorado Asthma Toolkit Program: a practice coaching intervention from the High Plains Research Network. J Am Board Fam Med 2011;24:240-8.

27. Nease DE, Jr., Nutting PA, Graham DG, Dickinson WP, Gallagher KM, Jeffcott-Pera M. Sustainability of depression care improvements: success of a practice change improvement collaborative. J Am Board Fam Med 2010;23:598-605.

28. Nease DE, Nutting PA, Dickinson WP, et al. Inducing sustainable improvement in depression care in primary care practices. Jt Comm J Qual Patient Saf 2008;34:247-55.

29. Institute of Medicine. Patients Charting the Course: Citizen Engagement in the Learning Health System Workshop Summary. Vol October 3. The National Academies Press, Washington, DC; 2011.
30. National Committee for Quality Assurance. PatientCentered Medical Home: recognition programs. Available from: http://www.ncqa.org/programs/ recognition/practices/patient-centered-medicalhome-pcmh. Accessed October 21, 2019.

31. Centers for Medicare \& Medicaid Services. CPC+ practice care delivery requirements. Available from: https://innovation.cms.gov/Files/x/cpcplus-practice caredlvreqs.pdf. Published 2015. Accessed October 21, 2019.

32. Sharma AE, Grumbach K. Engaging patients in primary care practice transformation: theory, evidence and practice. Fam Pract 2017;34:262-267.

33. Boivin A, Lehoux P, Lacombe R, Burgers J, Grol R. Involving patients in setting priorities for healthcare improvement: a cluster randomized trial. Implement Sci 2014;9:24.

34. Norman N, Bennett C, Cowart S, et al. Boot camp translation: a method for building a community of solution. J Am Board Fam Med 2013;26:254-63.

35. Zittleman L, Emsermann C, Dickinson M, et al. Increasing colon cancer testing in rural Colorado: evaluation of the exposure to a community-based awareness campaign. BMC Public Health 2009; 9:288.

36. Nease DE, Jr., Daly JM, Dickinson LM, et al. Impact of a boot camp translation intervention on self-management support in primary care. J Patient Cent Res Rev 2018;5:256-66.

37. Westfall JM, Zittleman L, Felzien $M$, et al. Reinventing the wheel of medical evidence: how the boot camp translation process is making gains. Health Aff (Millwood) 2016;35:613-8.

38. English AF, Dickinson LM, Zittleman L, et al. A community engagement method to design patient engagement materials for cardiovascular health. Ann Fam Med 2018;16:S58-S64.

39. Solberg LI. Improving medical practice: a conceptual framework. Ann Fam Med 2007;5:251-6.

40. Cohen D, McDaniel RRJ, Crabtree BF, et al. A practice change model for quality improvement in primary care practice. J Healthc Manag 2004;49:155-68; discussion 169-170.

41. Bodenheimer T, Ghorob A, Willard-Grace R, Grumbach K. The 10 building blocks of high-performing primary care. Ann Fam Med 2014; 12:166-71.

42. Hedeker D, Gibbons RD, Waternaux C. Sample size estimation for longitudinal designs with attrition. J Educ Behav Stat 1999;24:70-93.

43. DARTNet Institute. DARTNet website. Available from: http://www.dartnet.info/. Accessed October 21, 2019.

44. Centers for Disease Control and Prevention (CDC), National Heart Disease and Stroke Prevention Program. Best practices for cardiovascular disease prevention programs. Available from: https://www.cdc.gov/ 
dhdsp/pubs/guides/best-practices/index.htm. Accessed October 21, 2019.

45. eCQI Resource Center. Ischemic vascular disease (IVD): use of aspirin or another antiplatelet. Available from: https://ecqi.healthit.gov/ecqm/ep/ 2018/cms164v6. Published 2018. Accessed October $21,2019$.

46. eCQI Resource Center. Controlling high blood pressure. Available from: https://ecqi.healthit.gov/ ecqm/measures/cms165v6. Published 2018. Accessed October 21, 2019.

47. eCQI Resource Center. Statin therapy for the prevention and treatment of cardiovascular disease. Available from: https://ecqi.healthit.gov/ecqm/ep/ 2018/cms347v1. Published 2018. Accessed October $21,2019$.

48. eCQI Resource Center. Preventive care and screening: tobacco use: screening and cessation intervention. Available from: https://ecqi.healthit. gov/ecqm/measures/cms138v6. Published 2018. Accessed October 21, 2019.

49. Fairclough DL. Design and analysis of quality of life studies in clinical trials. New York, NY: Chapman \& Hall/CRC Press; 2010.

50. Diggle P, Kenward MG. Informative drop-out in longitudinal data analysis. Appl Stat 1994;43:49-93.

51. Hunger M, Doring A, Holle R. Longitudinal beta regression models for analyzing health-related quality of life scores over time. BMC Med Res Methodol 2012;12:144.

52. Ferrari SLP, Cribari-Neto F. Beta regression for modeling rates and proportions. J Appl Stat 2004;31:799-815.

53. Zimprich D. Modeling change in skewed variables using mixed beta regression models. Res Hum Dev 2010;7:9-26.

54. Hedeker D, Gibbons R. Longitudinal data analysis. Hoboken, New Jersey: Wiley \& Sons; 2006.

55. Kraemer HC, Wilson GT, Fairburn CG, Agras WS. Mediators and moderators of treatment effects in randomized clinical trials. Arch Gen Psychiatry 2002;59:877-83.

56. Dickinson LM, Beaty B, Fox C, et al. Pragmatic cluster randomized trials using covariate constrained randomization: a method for Practicebased Research Networks (PBRNs). J Am Board Fam Med 2015;28:663-72.

57. Cohen DJ, Dorr DA, Knierim K, et al. Primary care practices' abilities and challenges in using electronic health record data for quality improvement. Health Aff (Millwood) 2018;37:635-43.

58. Knierim KE, Hall TL, Dickinson LM, et al. Primary care practices' ability to report electronic clinical quality measures in the EvidenceNOW Southwest Initiative to improve heart health. JAMA Netw Open 2019;2:e198569. 